\title{
Lisa Golombek, Robert B. Mason, Patricia Proctor, Eileen Reilly. Persian Pottery in the First Global Age. The Sixteenth and Seventeenth Centuries.
}

\section{Sandra Aube}

\section{(2) OpenEdition \\ 1 Journals}

Édition électronique

URL : http://journals.openedition.org/abstractairanica/41242

DOI : $10.4000 /$ abstractairanica. 41242

ISSN : 1961-960X

Éditeur :

CNRS (UMR 7528 Mondes iraniens et indiens), Éditions de l'IFRI

Référence électronique

Sandra Aube, « Lisa Golombek, Robert B. Mason, Patricia Proctor, Eileen Reilly. Persian Pottery in the First Global Age. The Sixteenth and Seventeenth Centuries. », Abstracta Iranica [En ligne], Volume 34-35-36 | 2017, document 4, mis en ligne le 15 juillet 2016, consulté le 26 septembre 2020. URL : http://journals.openedition.org/abstractairanica/41242 ; DOI : https://doi.org/10.4000/

abstractairanica. 41242

Ce document a été généré automatiquement le 26 septembre 2020

Tous droits réservés 


\title{
Lisa Golombek, Robert B. Mason, Patricia Proctor, Eileen Reilly. Persian Pottery in the First Global Age. The Sixteenth and Seventeenth Centuries.
}

\author{
Sandra Aube
}

\section{RÉFÉRENCE}

Lisa Golombek, Robert B. Mason, Patricia Proctor, Eileen Reilly. Persian Pottery in the First Global Age. The Sixteenth and Seventeenth Centuries. Leyde/Boston, Brill, 2013, 502 p., 550 ill. (coll. Arts and Archaeology of the Islamic World)

1 À la suite de l'excellent Tamerlane's Tableware. A New Approach to Chinoiserie of Fifteenth and Sixteenth-Century Iran (avec Gauvin A. Bailey, Mazda Publishers, 1996), Lisa Golombek et Robert Mason livrent un nouvel opus de leur étude de la céramique iranienne, ici co-écrit avec Patricia Proctor et Eileen Reilly. À travers une étude du fonds du Royal Ontario Museum de Toronto (ROM) - largement enrichi de l'apport d'autres collections - et grâce aux données nouvelles livrées par les analyses pétrographiques, les AA. ouvrent une nouvelle page de l'histoire de la céramique en se consacrant ici aux XVI ${ }^{\mathrm{e}}$ et XVII ${ }^{\mathrm{e}}$ siècles safavides.

2 L'ouvrage s'organise en trois parties. Une première, composée de cinq chapitres, propose une étude de la société et de l'organisation de la production céramique (p. 11-209). En décryptant différents styles, les AA. proposent des éléments de datation nouveaux et un éclairage sur la production d'ateliers tels que Kermān et Mašhad. Les contacts avec la Chine y font l'objet d'une attention particulière, qu'il s'agisse des importations, de l'utilisation de modèles chinois, aussi bien que des productions locales 
(voir notamment le chapitre 3 «The Measure of Faithfulness: the Chinese Models for Safavid Blue-and-White», par P. Proctor, p. 123-166). On retiendra également la synthèse très attendue de Lisa Golombek sur la question des céramiques dites « de Kubacha ", avec la mise en évidence de l'atelier de Qumishe, à proximité d'Ispahan (chapitre 4, «The "Kubachi Problem" », p. 169-181).

3 La seconde partie se veut constituer un guide pour l'analyse des céramiques safavides (p. 210-278). Les trois chapitres qui composent cette section proposent une étude des motifs, des marques de potiers, puis des formes utilisées dans les céramiques safavides. La dernière section de ce livre offre enfin un catalogue des pièces safavides conservées au ROM (p. 279-429, 69 entrées).

4 L'ensemble est suivi d'annexes, parmi lesquelles on retiendra une très utile table des pièces datées (pièces de forme et céramique architecturale). Le volume est très largement illustré - bien que l'on pourra sans doute regretter, pour une publication de cette importance, la qualité toute relative de nombre d'illustrations. L'ensemble constitue à n'en pas douter un ouvrage de référence, très attendu, sur la céramique iranienne des $\mathrm{XVI}^{\mathrm{e}}$ et $\mathrm{XVII}^{\mathrm{e}}$ siècles.

\section{AUTEURS}

\section{SANDRA AUBE}

CNRS, Mondes iranien et indien, Paris 